\title{
Analysis, Optimisation and Simulation of Two Canceller Active Noise Control System
}

\author{
Branislav Vuksanovic ${ }^{*}$, Dragana Nikolic ${ }^{2}$ \\ 1 University of Portsmouth, School of Energy and Electronics, Portsmouth P013DJ, UK. \\ ${ }^{2}$ Grow Software Ltd, Fore Street, Moorgate, London EC2Y 9DT, UK. \\ * Corresponding author. Tel.: +44(0)2392842159; email: branislav.vuksanovic@port.ac.uk \\ Manuscript submitted October 15, 2018; accepted January 9, 2019. \\ doi: 10.17706/ijcee.2019.11.1.53-61
}

\begin{abstract}
This paper describes the analysis and simulation of a simple sound cancellation system consisting of a single point primary sound source and two cancelling sources. Conditions for the global sound attenuation along multiple axis in the system are derived and system behaviour and cancellation effects, studied through computer simulations. This study is aimed at understanding the physics of more complex active noise control systems (ANCS) consisting of higher number of secondary sources and error sensors in free field. Results provide a useful insight into ANCS performance and can therefore be used as a valuable reference point when studying and analysing the free field operation of a more complex, multi-canceller ANCS.
\end{abstract}

Key words: Active noise cancellation, multichannel control system, point monopole source, sound field optimization.

\section{Introduction}

Problem of acoustic noise is evident in everyday life as an increasing number of equipment produces sometimes excessive levels of acoustic noise [1]-[4]. Traditional approach of reduction of acoustic noise and unwanted sounds uses passive noise control techniques such are enclosures, barriers and silencers. Those methods, although relatively successful over a broad frequency range can be costly and ineffective for low frequency types of acoustic noise.

Aim of active noise control (ANC) is to reduce the unwanted and unpleasant sound by introducing the cancelling, or opposite sound - "antinoise" through a secondary sound source [5]. The principle is illustrated in Fig. 1. The idea of ANC, although intuitive and relatively simple can be difficult to implement in practice and the number of commercial active noise control systems (ANCS) available on the market today remains relatively low [6]. One of the problems successfully tackled by this technique is active control of low frequency plane sound waves propagating in ducts.

A single channel feedforward ANCS consists of two microphones and a cancelling loudspeaker as indicated in Fig. 1(b). The first microphone positioned close to the noise source senses the noise signal and the downstream microphone provides a noise cancellation error measurement. Cancelling loudspeaker or secondary sound source positioned between two microphones generates cancelling sound waves, i.e. "antinoise". Produced "antinoise" mixes with the original noise signal and assuming appropriately adjusted controller parameters; sound downstream of the error sensor gets cancelled or significantly attenuated.

The successful application of this system can be attributed to a relatively low spatial complexity of this 1-D 
problem. Control of 3-D sound in the free field is more complex problem and the simple, single channel active sound control system usually needs to be extended by adding more cancelling sources and error microphones thus creating a multichannel control system. By cancelling the sound at the error microphones distributed across the controlled area, the secondary sources create a zone of reduced noise through this region. This approach is also known as localised sound control. Different control algorithms can be used to implement this system in practice and generate the cancelling signal. One of the most common and effective approaches is the so-called "filtered-x least mean squares" method [7]. Filtered-x algorithm can be further extended to accommodate efficient multichannel ANCS [8].

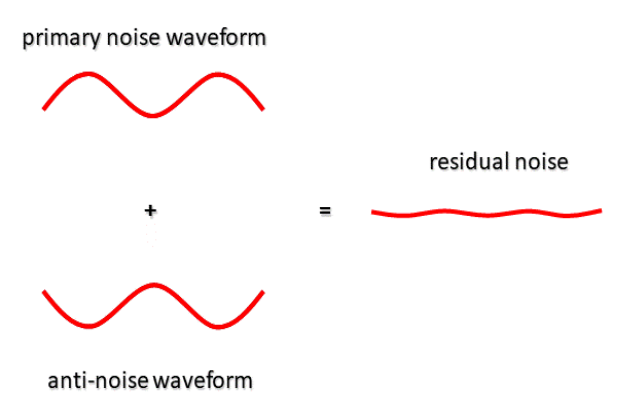

(a) sound cancellation principle

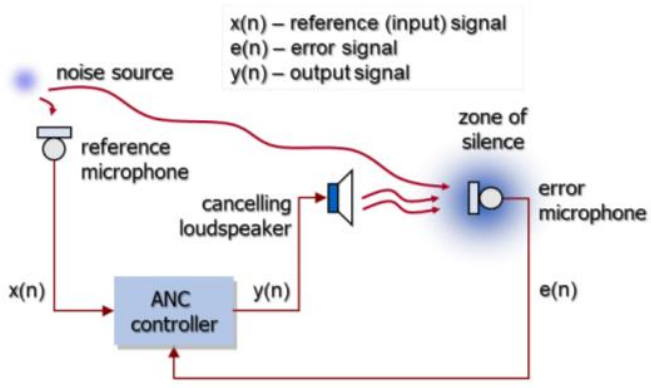

(b) active noise control system

Fig. 1. Active noise control essentials.

Complex, multi-canceller systems are usually analysed by deriving the conditions to attenuate the SPL locally, at a particular position of each error sensor in the system. The total sound pressure with secondary sources radiating according to this condition is then calculated and plotted for every point in the field so the global performance of this system can be studied and evaluated.

Better insight into physics of the 3-D sound cancelling system can be achieved by studying simpler acoustical systems first. Two-canceller system consisting of a single point primary source and two cancelling sources is analysed in this paper by deriving the conditions necessary to achieve global sound attenuation or at least attenuation along a number of axes. Results of this analysis can be used as a suitable reference for the performance of the more practical multi-canceller ANCS.

The approach to achieve sound attenuation along single and multiple axis in the system by minimising the sound pressure level along those axes is presented in this note. Calculated complex strengths of the cancelling point sound sources are then used in computer simulations to obtain sound pressure level at any point of interest in the system. Detailed derivation is shown in the Appendix.

The total power output of this system can also be minimized and conditions for the optimal system performance with respect to this measure obtained. Detailed derivation is omitted in this paper due to space limitation. This derivation will however be provided in the extended version of this paper.

\section{Two Canceller System}

The two-canceller model of the proposed ANCS with two, point secondary sources either side of the primary source is shown in Fig. 2. An ideal point sound source can be considered as a pulsating sphere that is infinitesimally small in size. The sphere is omni-directional, i.e. sound energy is emitted equally to all directions. The complex sound pressure radiated from a point monopole source and denoted as $p(r)$ can be expressed as [9]:

$$
p(r)=\frac{j \omega \rho_{0} e^{-j k r}}{4 \pi r} q=z(r) q
$$




$$
z(r)=\frac{j \omega \rho_{0} e^{-j k r}}{4 \pi r}
$$

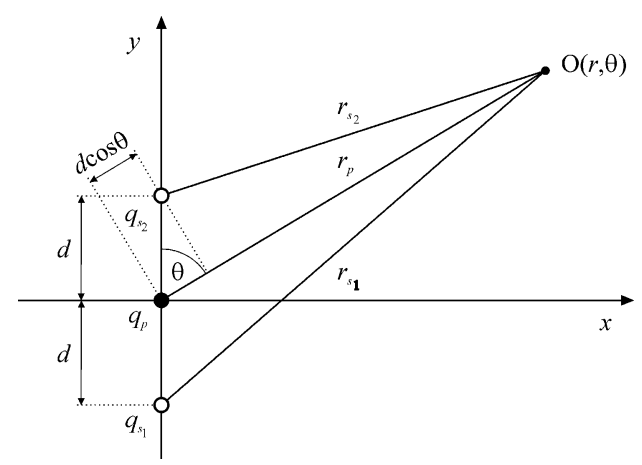

Fig. 2. Primary and secondary point monopole sources separated by a distance $d$.

where $q$ is the complex source strength, $r$ is the distance from the point source to the observer point, $\omega$ is angular frequency, $\rho_{0}$ is the density of the propagation medium, and $z(r)$ is the acoustic transfer impedance (propagation coefficient). $k$ is the so-called wave number, reciprocal parameter to wavelength $\lambda$, thus indicating the number of waves per unit distance.

The overall complex pressure at some observation point $\mathrm{O}(r, \theta)$, at an angle $\theta$ and a distance $r$ from the primary source, can be calculated using the principle of superposition as a sum of pressures produced by each source independently:

$$
\begin{aligned}
p(r, \theta) & =p\left(r_{p}, \theta\right)+p\left(r_{s_{1}}, \theta\right)+p\left(r_{s_{2}}, \theta\right) \\
& =z\left(r_{p}\right) q_{p}+z\left(r_{s_{1}}\right) q_{s_{1}}+z\left(r_{s_{2}}\right) q_{s_{2}} \\
& =\frac{j \omega \rho_{0}}{4 \pi}\left(\frac{e^{-j k r_{p}}}{r_{p}} q_{p}+\frac{e^{-j k k_{s_{1}}}}{r_{s_{1}}} q_{s_{1}}+\frac{e^{-j k r_{s_{2}}}}{r_{s_{2}}} q_{s_{2}}\right)
\end{aligned}
$$

Here $q_{p}$ is the primary source complex sound strength, $q_{s_{1}}$ and $q_{s_{2}}$ are the secondary sources' complex sound strengths, $r_{p}$ is the radial distance from the primary source and $r_{s_{1}}$ and $r_{s_{2}}$ are the radial distances from the secondary sources to the observation point respectively. This expression can be simplified using the far field approximations for the radial distances where $r_{s_{1}} \approx r_{p}+d \cos \theta$ and $r_{s_{2}} \approx r_{p}-d \cos \theta$.

For the values appearing in the denominator in (3), a further approximation $r_{s_{1}} \approx r_{s_{2}} \approx r_{p} \approx r$ can also be made. Assuming that the secondary source strengths are equal, $q_{s_{1}}=q_{s_{2}}=q_{s}$, (3) can be rewritten as:

$$
p(r, \theta)=z(r)\left(q_{p}+2 q_{s} \cos (k d \cos \theta)\right)
$$

Placing the error sensor at an angle $\theta_{0}$ from the source arrangement and calculating the complex sound strength of the secondary sources necessary to achieve $p\left(r, \theta_{0}\right)=0$ will result in the condition for the local attenuation of the sound pressure derived in (4). To ensure that zero pressure is produced in the far field at a particular angular position specified by $\theta_{0}$, the secondary source strengths therefore need to be:

$$
q_{s}=-\frac{q_{p}}{2 \cos \left(k d \cos \theta_{0}\right)}
$$

Having defined $\theta_{0}$ such that far field pressure in that direction is zero $p\left(r, \theta_{0}\right)=0$, the overall sound 
pressure at any other angle $\theta$ can be derived by substituting (5) into (4):

$$
p(r, \theta)=z(r) q_{p}\left(1-\frac{\cos (k d \cos \theta)}{\cos \left(k d \cos \theta_{0}\right)}\right)
$$

The square of the modulus of the relative far field pressures is given by:

$$
\left|\frac{p(r, \theta)}{p_{p}(r, \theta)}\right|^{2}=\left[1-\frac{\cos (k d \cos \theta)}{\cos \left(k d \cos \theta_{0}\right)}\right]^{2}
$$

where $p_{p}(r, \theta)=z(r) q_{p}$ is the primary complex sound pressure due to primary source acting alone.

Polar plots of this modulus squared for separation distances $d$ of $5 \lambda / 36, \lambda / 6$ and $\lambda$ and $\theta_{0}=0^{\circ}$ are shown in Fig. 3. Obviously, it is possible to produce zero pressure in the far field of this three-point-source arrangement at any angular position, but this destructive interference at one particular location might be accompanied by a constructive interference at some other locations in the field.

For the successful global control of sound fields, the separation between primary and secondary sources should be much less than the wavelength of the radiated sound. Depending on the selected direction to be controlled, the critical value for separation distance that provides attenuation in all directions is found to be $d=\lambda / 6$ for $\theta_{0}=0^{\circ}$ and $d=\lambda / 4$ for $\theta_{0}=90^{\circ}$.

A general condition for producing attenuation of the primary field at all angular locations $\theta$, can be derived from (7). In order to achieve $|p(r, \theta)|<\left|p_{p}(r, \theta)\right|$, the following condition needs to be satisfied:

$$
\left|1-\frac{\cos (k d \cos \theta)}{\cos \left(k d \cos \theta_{0}\right)}\right|<1
$$

The ratio $\frac{\cos (k d \cos \theta)}{\cos \left(k d \cos \theta_{0}\right)}$ achieves a maximum of $\frac{1}{\cos k d}$ when $\theta_{0}=0^{\circ}$ and $\theta=90^{\circ}$, so it follows that:

$$
0<\frac{\cos (k d \cos \theta)}{\cos \left(k d \cos \theta_{0}\right)}<\frac{1}{\cos k d}<2 .
$$

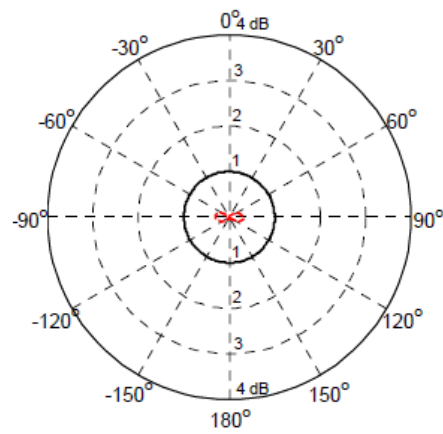

(a) $d=5 \lambda / 36$

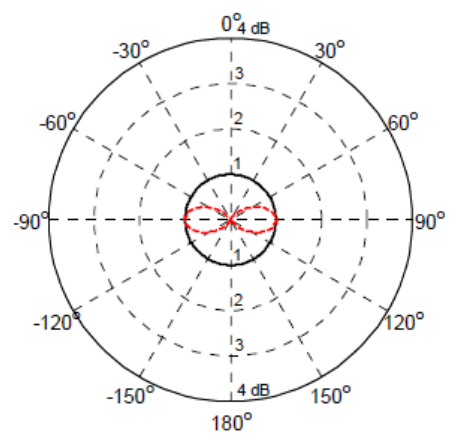

(b) $d=\lambda / 6$

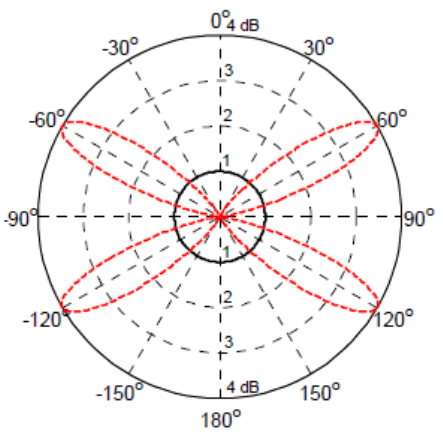

(c) $d=\lambda$

Fig. 3. The modulus squared of far field sound pressure for $\theta_{0}=0^{\circ}$ (primary pressure - solid line, overall pressure - dashed line).

To satisfy the above condition, the separation distance $d$ needs to satisfy $k d<\lambda / 3$ i.e. $d<\lambda / 6$. In general, for producing attenuation at all angular directions $\theta$, irrespective of the choice of $\theta_{0}, d$ must be less than $\lambda / 6$. The performance of this arrangement can be studied by plotting the achieved attenuation level - difference in $\mathrm{dBs}$ between the SPL generated by the primary source radiating on its own (the uncancelled field) and the 
SPL with all three sound sources radiating together with the complex strengths of the secondary sources determined by (5) (the cancelled field).

The frequency of the primary source is chosen to be $500 \mathrm{~Hz}$. The distance between the primary and secondary sources is $\lambda / 8$. Three results for error sensor angles $\left(0^{\circ}, 45^{\circ}\right.$ and $\left.90^{\circ}\right)$ are shown in Fig. 4 . The angular positions of the error sensors obviously determine the location of the zones with higher attenuation levels, but the global SPL reduction is also achieved because $d<\lambda / 6$.

To improve the global performance of the system for larger primary-secondary source distances where $d>\lambda / 6$, the secondary source strengths can be optimised to reduce the SPL along more than one axis in the system. Minimising the sum of the sound fields generated independently by each point source, the secondary source strengths that minimise sound pressure in $M$ directions (each defined by an angle $\theta_{m}$ ) is obtained:

$$
q_{s}=-\frac{\sum_{m=1}^{M} \cos \left(k d \cos \theta_{m}\right)}{2 \sum_{m=1}^{M} \cos ^{2}\left(k d \cos \theta_{m}\right)} q_{p}
$$
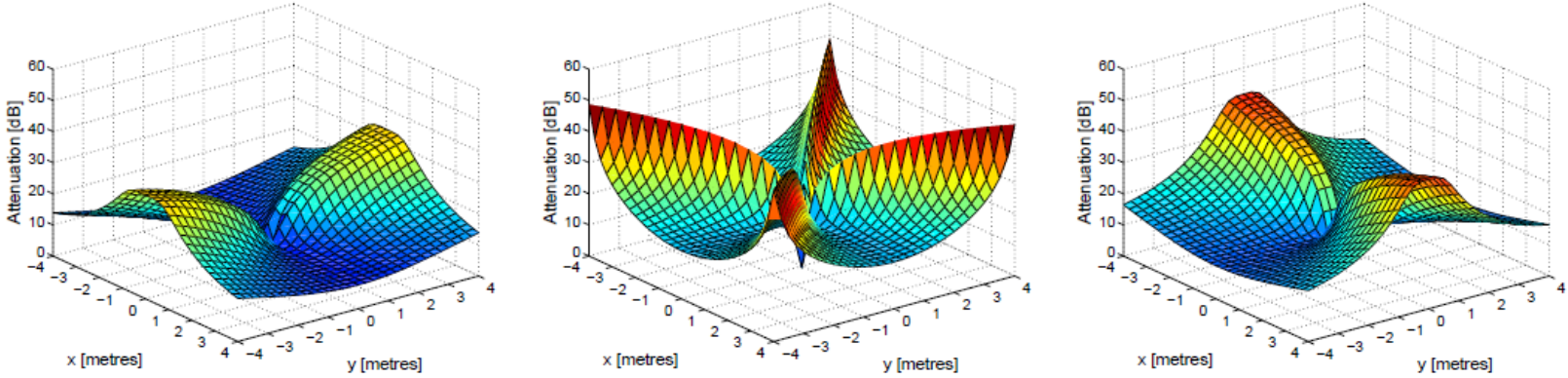

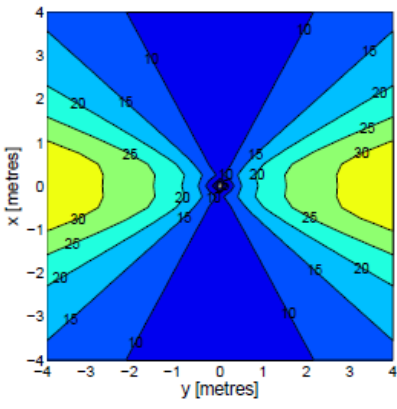

(a)

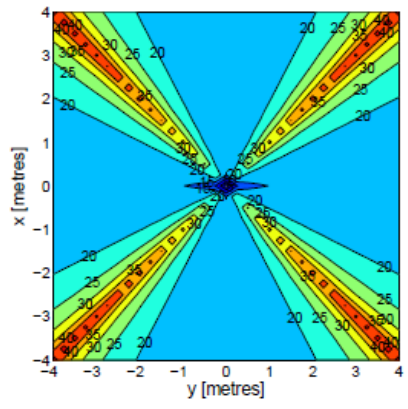

(b)

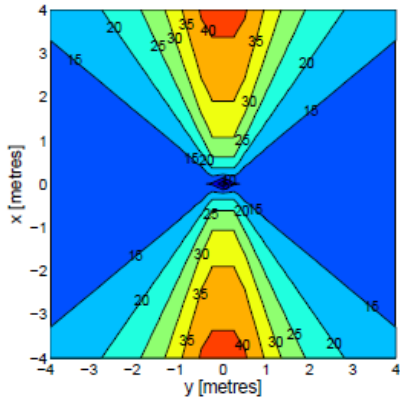

(c)

Fig. 4. Attenuation level measured in the $x y$ observer plane at frequency $f=500 \mathrm{~Hz}$ and separation distance $d=\lambda / 8$.

where $\theta_{m}$ is in range $\left[0,90^{\circ}\right]$ as a result of the system symmetry. Previously obtained (5) therefore represents the special case of (10) where $M=1$.

Using this expression for the optimal secondary source strengths, attenuation plots equivalent to ones produced in Fig. 4 have been generated for the case of 2 secondary sources, distance $d=\lambda / 3$ between the sources and $M=1,2$ and 3 control angles respectively. These results are shown in Fig. 5. Fig. 5(a), plotted for the case of $M=1$, demonstrates that global attenuation for this setup is not possible since $d=\lambda / 3>\lambda / 6$. Although $15-20 \mathrm{~dB}$ attenuation has been achieved for angles close to control angle of $\theta_{0}=0^{\circ}$, further away from this zone, for angles close to $90^{\circ}$, negative attenuation, i.e. the actual increase in the SPL, has been recorded. To improve the performance of this system secondary source strengths have been modified according to (10) 
using two additional control angles $\theta_{1}=30^{\circ}$ and $\theta_{2}=60^{\circ}$. By comparing plots in Fig. 4 , it can be seen that no significant increase in the level of attenuation has been achieved by attempting to reduce the SPL at more than one angular direction. The described two-canceller system is not capable of reducing the SPL significantly along the specific control angles. For the case of two control angles $\theta_{0}=0^{\circ}$ and $\theta_{1}=30^{\circ}$ (Fig. 5(b)), maximum attenuation is obtained along the angle of approximately $15^{\circ}$ ! For the case of three control angles (Fig. 5(c)) achieved attenuation is negligible, close to $0 \mathrm{~dB}$ across the whole region.

\section{Conclusions}

This paper describes the behaviour of a simple ANCS consisting of a single point primary sound source and two cancelling sources. Conditions for the sound attenuation along multiple axis in the system have been derived and the system behaviour studied through computer simulations.

Additional conditions required to minimise total power output of the two-secondary and one primary sound source arrangement can also be established. Using this approach, the total power output is reduced for any choice of $\theta_{0}$ if the separation distance between the primary and secondary sources is smaller than about one ninth of the wavelength. Details of this derivation are omitted due to space limitation.

However, the obtained results indicate that the satisfactory sound attenuation would usually be difficult to achieve with a simple two-canceller system. To improve the performance of the ANCS, it is therefore necessary to consider the system with more than two secondary sources. Derivation of the equations for the sound attenuation along multiple axis in the system would be too complex for the multi-canceller ANCS. For practical applications, rather than trying to reduce SPL along the system axis, it is actually easier and more suitable to monitor the system performance at the particular discrete points in the field. The multi-canceller system aimed at reducing the SPL at each sensor location in the field will be considered in the extension of this work. The optimal secondary source strengths that can reduce the SPL at sensor positions of such a system will be derived and analysed and a simple mathematical model to simulate such system will be presented in order to establish the achievable level of sound attenuation using general multi-canceller type ANCS.

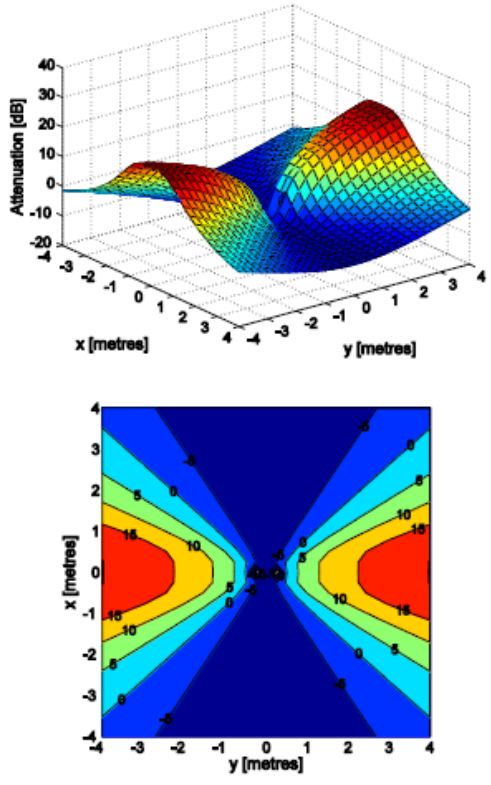

(a)

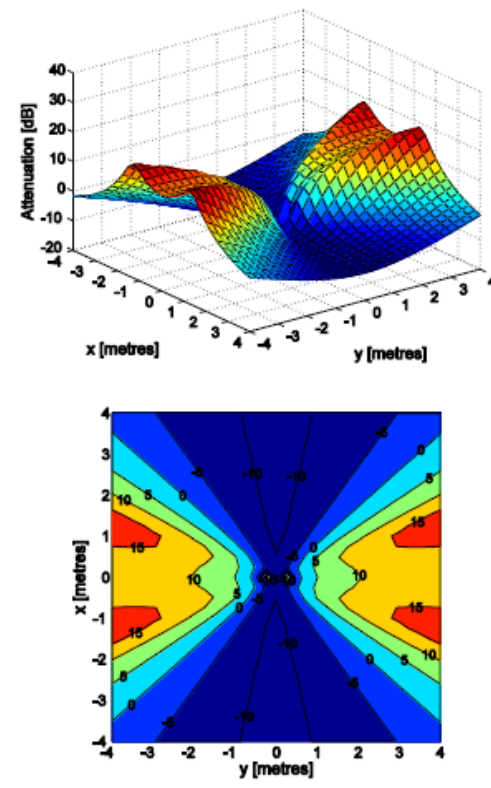

(b)

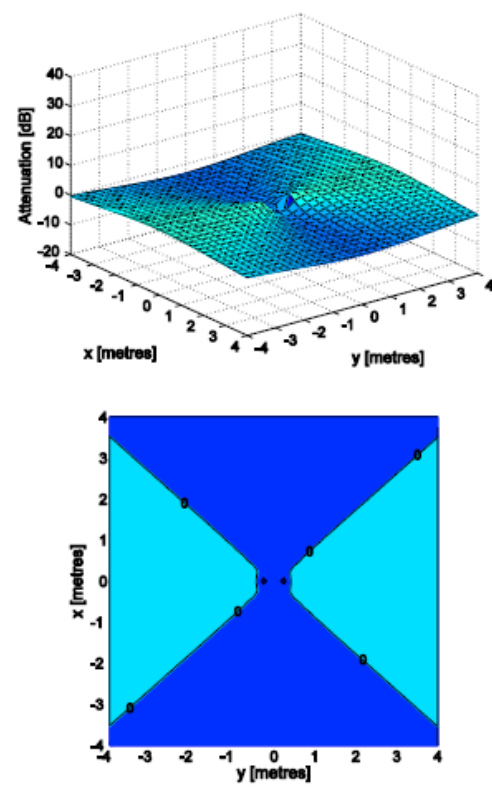

(c)

Fig. 5. Attenuation level measured in the xy observer plane at frequency $f=500 \mathrm{~Hz}$ and separation distance $d=\lambda / 3$ for varying number of sensor angle. 


\section{Appendix}

\section{Minimisation of the Sound Pressure Level for the Two-Canceller System along $M$ System Axes}

Here, we show how a sound pressure can be minimised for a single primary and two secondary sources arrangement along $M$ angular positions in the field.

For this purpose, $M$-element vector $\mathbf{p}$ containing the complex far field pressures at $M$ positions along angles $\theta_{1}, \theta_{2}, \ldots \theta_{M}$ is defined:

$$
\mathbf{p}^{\mathbf{T}}=\left[\begin{array}{llll}
p\left(r, \theta_{1}\right) & p\left(r, \theta_{2}\right) & \ldots & p\left(r, \theta_{M}\right)
\end{array}\right]
$$

Total complex sound pressure in the field can be calculated as a superposition of the fields from the primary and two secondary sources. In the case when the secondary source strengths are equal, $q_{s_{1}}=q_{s_{2}}=q_{s}, \mathbf{p}$ is given by:

$$
\mathbf{p}=\mathbf{z}_{\mathbf{p}} q_{p}+\left(\mathbf{z}_{\mathbf{s}_{1}}+\mathbf{z}_{\mathbf{s}_{2}}\right) q_{s}
$$

where $\mathbf{z}_{p}, \mathbf{z}_{s_{1}}$ and $\mathbf{z}_{s_{2}}$ are vectors of the complex acoustic propagation coefficients (transfer impedances) defined as:

$$
\begin{aligned}
& \mathbf{z}_{\mathbf{p}}^{\mathbf{T}}=\left[\begin{array}{llll}
z\left(r_{p_{1}}\right) & z\left(r_{p_{2}}\right) & \ldots & z\left(r_{p_{M}}\right)
\end{array}\right] \\
& \mathbf{z}_{\mathbf{s}_{1}}^{\mathbf{T}}=\left[\begin{array}{llll}
z\left(r_{s_{11}}\right) & z\left(r_{s_{12}}\right) & \ldots & z\left(r_{s_{1 M}}\right)
\end{array}\right] \\
& \mathbf{z}_{\mathbf{s}_{2}}^{\mathbf{T}}=\left[\begin{array}{llll}
z\left(r_{s_{21}}\right) & z\left(r_{s_{22}}\right) & \ldots & z\left(r_{s_{2 M}}\right)
\end{array}\right]
\end{aligned}
$$

The cost function to be minimised for global sound reduction is defined as the sum of the modulus squared of the pressures at all $M$ sensor positions:

$$
J_{p}=\mathbf{p}^{\mathbf{H}} \mathbf{p}=\sum_{m=1}^{M}\left|p\left(r, \theta_{m}\right)\right|^{2}
$$

By substituting (2) into (6), the cost function can be expressed in the following form:

$$
\begin{aligned}
J_{p} & =q_{s}^{*}\left(\mathbf{z}_{\mathbf{s}_{1}}+\mathbf{z}_{\mathbf{s}_{2}}\right)^{\mathbf{H}}\left(\mathbf{z}_{\mathbf{s}_{1}}+\mathbf{z}_{\mathbf{s}_{2}}\right) q_{s}+q_{s}^{*}\left(\mathbf{z}_{\mathbf{s}_{1}}+\mathbf{z}_{\mathbf{s}_{2}}\right)^{\mathbf{H}} \mathbf{z}_{\mathbf{p}} q_{p} \\
& +q_{p}^{*} \mathbf{z}_{\mathbf{p}}^{\mathbf{H}}\left(\mathbf{z}_{\mathbf{s}_{1}}+\mathbf{z}_{\mathbf{s}_{2}}\right) q_{s}+q_{p}^{*} \mathbf{z}_{\mathbf{p}}^{\mathbf{H}} \mathbf{z}_{\mathbf{p}} q_{p}
\end{aligned}
$$

It can be proved that the optimal set of secondary source strengths used to minimise the given cost function is given by:

$$
q_{\text {sopt }}=-\frac{\left(\mathbf{z}_{\mathbf{s}_{1}}+\mathbf{z}_{\mathbf{s}_{2}}\right)^{\mathbf{H}} \mathbf{z}_{\mathbf{p}}}{\left(\mathbf{z}_{\mathbf{s}_{1}}+\mathbf{z}_{\mathbf{s}_{2}}\right)^{\mathbf{H}}\left(\mathbf{z}_{\mathbf{s}_{1}}+\mathbf{z}_{\mathrm{s}_{2}}\right)} q_{p}
$$

Since:

$$
\mathbf{z}_{\mathbf{s}_{1}}^{\mathbf{H}} \mathbf{z}_{\mathbf{p}}=\left(\frac{\omega \rho_{0}}{4 \pi}\right)^{2} \sum_{m=1}^{M} \frac{e^{-j k\left(r_{p_{m}}-r_{s_{1 m}}\right)}}{r_{s_{1 m}} r_{p_{m}}} \quad \text { and } \quad \mathbf{z}_{\mathbf{s}_{2}}^{\mathbf{H}} \mathbf{z}_{\mathbf{p}}=\left(\frac{\omega \rho_{0}}{4 \pi}\right)^{2} \sum_{m=1}^{M} \frac{e^{-j k\left(r_{r_{m}}-r_{r_{2 m}}\right)}}{r_{s_{2 m}} r_{p_{m}}}
$$

Using far field assumptions, the term in numerator of (8) can be written as: 


$$
\begin{aligned}
\left(\mathbf{z}_{\mathbf{s}_{1}}+\mathbf{z}_{\mathbf{s}_{2}}\right)^{\mathbf{H}} \mathbf{z}_{\mathbf{p}} & =\left(\frac{\omega \rho_{0}}{4 \pi r}\right)^{2} \sum_{m=1}^{M}\left(e^{j k d \cos \theta_{m}}+e^{-j k d \cos \theta_{m}}\right) \\
& =2\left(\frac{\omega \rho_{0}}{4 \pi r}\right)^{2} \sum_{m=1}^{M} \cos \left(k d \cos \theta_{m}\right)
\end{aligned}
$$

In order to calculate the form of the denominator in (8), the $m$-th element of the vector $\mathbf{z}_{\mathbf{s}_{1}}+\mathbf{z}_{\mathbf{s}_{2}}$ is derived as:

$$
\begin{aligned}
\left(\mathbf{z}_{\mathbf{s}_{1}}+\mathbf{z}_{\mathbf{s}_{2}}\right)_{m} & =\frac{j \omega \rho_{0}}{4 \pi}\left(\frac{e^{-j k r_{1 / m}}}{r_{s_{1 m}}}+\frac{e^{j k r_{s_{2 m}}}}{r_{s_{2 m}}}\right) \\
& =2 \frac{j \omega \rho_{0} e^{-j k r}}{4 \pi r} \cos \left(k d \cos \theta_{m}\right)
\end{aligned}
$$

It follows that the denominator of (8) can be written as:

$$
\begin{aligned}
\left(\mathbf{z}_{\mathbf{s}_{1}}+\mathbf{z}_{\mathbf{s}_{2}}\right)^{\mathbf{H}}\left(\mathbf{z}_{\mathbf{s}_{1}}+\mathbf{z}_{\mathrm{s}_{2}}\right) & =\sum_{m=1}^{M}\left|\left(\mathbf{z}_{\mathbf{s}_{1}}+\mathbf{z}_{\mathbf{s}_{2}}\right)_{m}\right|^{2} \\
& =4\left(\frac{\omega \rho_{0}}{4 \pi r}\right)^{2} \sum_{m=1}^{M} \cos ^{2}\left(k d \cos \theta_{m}\right)
\end{aligned}
$$

Finally, the optimal solution for secondary source strengths that will minimise sound pressure in all $M$ directions (each defined by angle $\theta_{m}$ ) is given by:

$$
q_{s}=-\frac{\sum_{m=1}^{M} \cos \left(k d \cos \theta_{m}\right)}{2 \sum_{m=1}^{M} \cos ^{2}\left(k d \cos \theta_{m}\right)} q_{p}
$$

\section{References}

[1] Pal, D., \& Bhattacharya, D. (2012). Effect of road traffic noise pollution on human work efficiency in government offices, private organizations, and commercial business centres in Agartala city using fuzzy expert system: A case study. Advances in Fuzzy Systems, 2012.

[2] Savale, P. A. (2014). Effect of noise pollution on human being: Its prevention and control Journal of Environmental Research and Development, 8(4), 1026-1036.

[3] Giusti, G. D., \& Piergentili, F. (2009). Noise in intensive care unit A summary review. Iranian Journal of Otorhinolaryngology, 7.

[4] Darbyshire, J. L. (2016). Excessive noise in intensive care units. BMJ, 353, 1-2.

[5] Kuo, S., \& Morgan, D. R. (1999). Active noise control: A tutorial review. Proceedings of the IEEE: Vol 87.

[6] Kuo, S., Kuo, K., \& Gan, W. S. (2010). Active noise control: Open problems and challenges. Proceedings of the 2010 International Conference on Green Circuits and Systems.

[7] Ardekani, I. T., \& Abdulla, W. H. (2011). FxLMS-based active noise control: A quick review. Proceedings of the APSIPA ASC: Vol 1. (pp. 1-11).

[8] Nikolić, D., Vuksanović, B., \& Parchizadeh, H. (2009, September-October). Multichannel filtered inverse LMS algorithm for ANC: Laboratory implementation and testing. Acta Acoustica United with Acoustica, 95(5), 767-962.

[9] Nelson, P. A., \& Elliott, S. J. (1991). Active Control of Sound. Academic Press. 


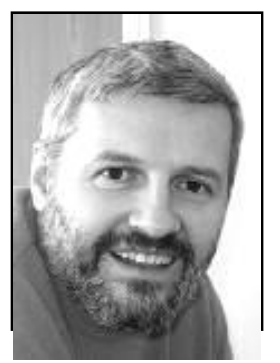

Branislav Vuksanovic has graduated from the University of Belgrade, Serbia with degree in electrical and power engineering. He holds an MSc degree in measurement and instrumentation from South Bank University, London and a PhD in active noise control from the University of Huddersfield, UK.

Previously, he worked as a project engineer for Croatian Electricity Board in Osijek, Croatia. During his academic career he worked as a research fellow at Sheffield and Birmingham Universities and a lecturer at the University of Derby. He has published papers in the field of active noise control, biomedical signal processing and pattern recognition for intrusion detection and knowledge-based authentication. Currently, he works as a senior lecturer at the University of Portsmouth.

Dr. Branislav Vuksanovic is a member of IET. His current research interests are in the application of pattern recognition techniques for power systems and analysis of ground penetrating radar and ECG data.

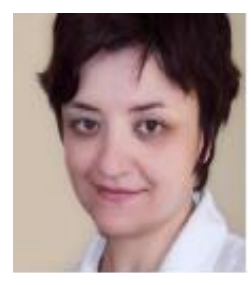

Dragana Nikolic has graduated from the University of Belgrade, Serbia with degree in electrical and electronic engineering. After her graduation, she worked at the University of Belgrade, Faculty of Technology as a research and teaching assistant. She has completed her Ph.D studies at the University of Portsmouth, UK in the area of active acoustic cancellation and adaptive filtering. Following this, she has worked as a research fellow in the biomedical signal processing at the University of Southampton, Institute of Sound and Vibration for a number of years and has acted as an editorial assistant for two journals in that period - Journal of Biomedical Signal Processing and Control and Journal of Sound and Vibration.

Her research interests and expertise are in the areas of signal processing and machine learning including bioacoustics and biomedical data analysis and processing. She is currently working as a data analyst in the city of London. 